\title{
The design of a novel structural four-beams-bossed- membrane (FBBM) piezoresistive pressure sensor
}

\author{
Chuang Li, Francisco Cordovilla, José L. Ocaña
}

\section{INTRODUCTION}

MEMS-based piezoresistive pressure sensors are widely employed in different applications due to advantages such as small scale, direct signal transduction mechanism, and mature fabrication [1]. Recently, the development towards biomedical, microphones and atomic force microscope probes has further expanded its application of piezoresistive sensing [2]. However, the trade-off between sensitivity and linearity of the sensor is always irreconcilable, especially in the field of micro-pressure measurements [3]. For the same membrane thickness, larger membranes offer high sensitivity with poor linearity and vice versa are true for smaller membranes. Therefore, alleviating the contradiction between the sensitivity and linearity is the key to improve the accuracy of the sensor.

In this work, a novel four-beams-bossed-membrane (FBBM) structure piezoresistive pressure sensor was reported. Through introducing beams into the membrane, the stress concentration regions (SCRs) were expected to be appeared. Piezoresistors were placed on the SCRs and the piezoresistive sensitivity would be improved with a small deflection of the membrane [4]. In addition, the bossed-membrane could play a role in reducing the deflection, and consequently, lower pressure nonlinearity (PNL) [5]. In some degree the chip geometry determines the performance of the sensor [6]. Thus, the geometry design and

optimization of a sensor membrane are significant. To improve the properties of the pressure sensor, the mechanical performance and relative equations for accurate design and fabrication were fully studied. The fitting equations were presented based on the finite element analysis (FEA) using COMSOL Multiphysics software and curve-fitting method by Origin. Consequently, a series of optimized dimension variables of the sensor membrane were determined. On the basis of the optimized model, the static characteristics of the sensor were also studied. The MEMS bulk-micromachining and anodic bonding technologies were chosen to realize the sensor.

\section{GEOMETRY DESIGN}

\section{A. Structural design}

A novel FBBM structural chip featuring a square bossed membrane with four short beams is designed. The sensor chip is fabricated with $\mathrm{N}$-type silicon wafer with the thickness of $300 \pm 5 \mu \mathrm{m}$. The FBBM structure is constructed in detail as shown in Fig. 1a, where $L$ is the effective side length of the membrane, $a, b$ and $h$ are the length, width and thickness of each beam, respectively. The rearview of the FBBM structure is presented in Fig. 1b, where $H$ represents the thickness of membrane, $w$ and $t$ are the side length and thickness of the central mass. The dorsal cavity fabricated by wet etching provides space for deformation of the membrane, and a $54.7^{\circ}$ inclined wall is appeared due to anisotropy of single crystal silicon [7]. In this model, SCRs are expected to be located at the joint between each beam and membrane pedestal side. Then, the strain energy is strictly limited in a narrow area on the surface of the beams and the energy is not dispersed easily outside the SCRs. Moreover, the central mass could play an important role in local stiffening, it is beneficial to limit a large deflection effect and thereby reduces the pressure nonlinearity error.

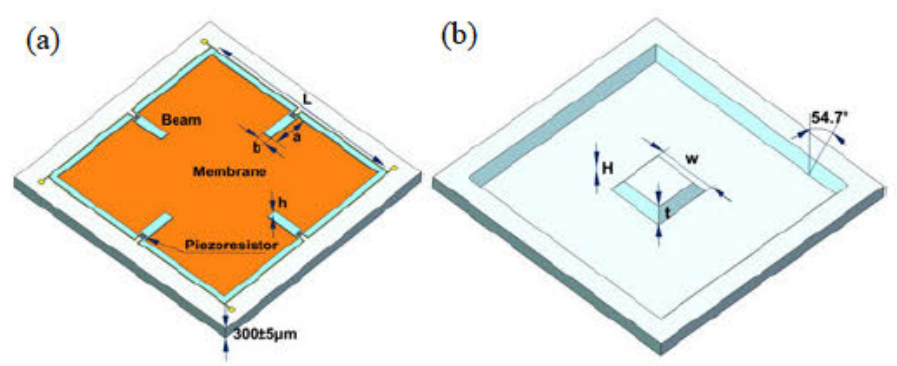

Fig. 1. (a) Sketch of the FBBM structure in the front view; (b) Rearview sketch of the FBBM structure. 


\section{B. Simulation analysis}

The performances of the proposed FBBM sensor chip are calculated by non-linear static analysis and model analysis by FEA. It can be seen that the maximum membrane deflection is happened at the center of the chip as shown in Fig. 2a. Its value is $<4 \mu \mathrm{m}$ which is under $1 / 5$ thickness of the membrane. Based on the small deformation theory introduced above, the central mass pattern can make a contribution to the stiffness change, it can decrease the pressure nonlinearity to smaller than $1.0 \% \mathrm{FSS}$ by stiffening the membrane. Besides, Fig. $2 \mathrm{~b}$ reflects the stress distribution under $5 \mathrm{kPa}$ uniform pressure. In accordance with the previous research, the strain energy is mainly concentrated at the SCRs which locate at the edge of the beams on the membrane, as indicated by the red area. This SCRs condition benefits from the novel stress concentration enhanced structure. It can be observed that the maximum stress appears at SCRs, and the stress in the region of the central mass is almost equal to zero. Owning to the stiffness change created by the novel beams structure combined with a central mass, the strain energy is strictly limited in a narrow area. So the strain energy utilized by four piezoresistors is more than flat membrane, which makes it possible to obtain high sensitivity. By this means, the FBBM structural sensor will be a suitable choice for improving sensitivity and linearity simultaneously.

(a)

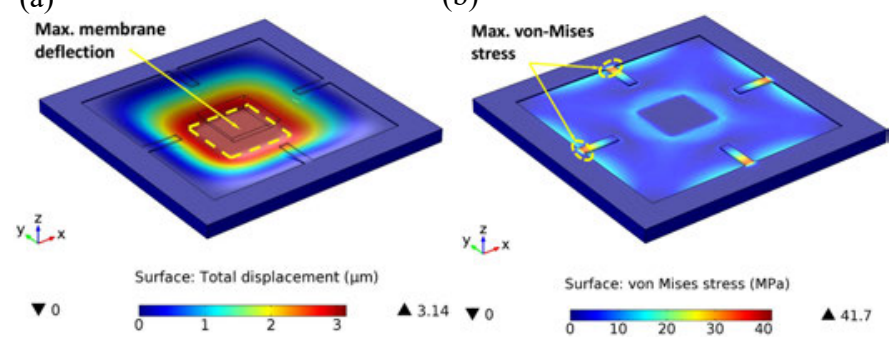

Fig. 2. Simulations for the membrane of sensor: (a) Max. membrane deflection; (b) Max. von-Mises stress.

\section{Geometry optimization}

The sensitivity of a pressure sensor depends on the initial resistance of the piezoresistors, which in turn is dependent on the geometry dimensions of the chip. In order to determine and optimize the structural dimensions, it is necessary to derive the relationship between the structure and performance for FBBM designing. The simulation results of the maximum stress and deflection with different structural dimension variables are presented below. According to the formulas of traditional Ctype membrane structure, the maximum stress and deflection of the membrane are the power functions of each single structural dimension variable [8]. Theoretically, the function relationship between FBBM structure and C-type should be similar. Based on previous study [4], Young's elastic modulus $E$ had almost no influence on the maximum stress $\sigma$, but revealed the inverse proportional relationship to the maximum deflection $\omega$. As a result, the formulas of FBBM are expressed as follows:

$$
\begin{gathered}
\sigma=Q_{1} \cdot P \cdot L^{i 1} \cdot H^{k 1} \cdot a^{m 1} \cdot b^{n 1} \cdot h^{r 1} \cdot w^{s 1} \cdot t^{z 1} \\
w=Q_{2} \cdot P \cdot E^{-1} \cdot L^{i 2} \cdot H^{k 2} \cdot a^{m 2} \cdot b^{n 2} \cdot h^{r 2} \cdot w^{s 2} \cdot t^{z 2}
\end{gathered}
$$

where $L, H, a, b, h, w, t$ are the structural dimensions as shown in Fig. $1 ; \sigma$ and $\omega$ are the maximum mechanical stress and maximum deflection of the membrane under pressure; and $Q_{l}$, $Q_{2}, i_{1}, i_{2}, k_{1}, k_{2}, m_{1}, m_{2}, n_{1}, n_{2}, r_{1}, r_{2}, s_{1}, s_{2}, z_{1}, z_{2}$ are the coefficients. To derive the coefficients in Eq. (1) and Eq. (2), each single dimension variable should be discussed separately. It means that other variables should be seen as constants when one variable is studied. Therefore, the relationship between the sensor chip performance and membrane length satisfies Eq. (3) and Eq. (4):

$$
\begin{aligned}
& \sigma=Q_{1 l} \cdot L^{i 1} \\
& \omega=Q_{2 l} \cdot L^{i 2}
\end{aligned}
$$

where $Q_{l l}, Q_{2 l}$ are the sub-coefficients of $L$. A series of $\sigma$ and $\omega$ are calculated by COMSOL numerical simulation when the membrane length is varied. Then, the equations are derived as follows by curve-fitting method.

$$
\begin{gathered}
\sigma=8.16818 \times 10^{-10} L^{2.93978} \\
\omega=8.93579 \times 10^{-19} L^{5.07637}
\end{gathered}
$$

Utilize the same analytical manner, the function relationships respect to other dimension variables can be achieved. Then, by combining each single dimension variable from the equation, the particular Eq. (1) and Eq. (2) are determined. The relative main equations can be derived as Eq. (7) and Eq. (8):

$$
\begin{gathered}
\sigma=Q_{1} \frac{P L^{293978} a^{031799}}{H^{190014} b^{03945} h^{040149} w^{021938} t^{002068}} \\
w=Q_{2} \frac{P L^{507637}}{E H^{235753} a^{016714} b^{02673} h^{05443} w^{073648} t^{009719}}
\end{gathered}
$$

From Eq. (7) to Eq. (8), it can be concluded that with the increase of the $L$, the $\sigma$ and $\omega$ both experience a rise, namely, improves sensitivity, but decreases linearity. Besides, the impacts of the $H, b, h, w$ and $t$ on the sensitivity and linearity are the same except $a$. The beam length is the only dimension variable which is in direct proportional to the stress and inverse proportional to the deflection. It is indicated that the sensitivity and linearity can be improved synchronously when the beam length is chosen appropriately. So it is reasonable to believe the contradiction between the sensitivity and linearity can be eliminated if the suitable dimension variables are determined.

The impacts of structural dimension variables on the mechanical stress are presented in Fig. 3. It can be regarded as an estimation for the performance of the sensor, as each dimension variable has an effect on the stress. For clear observation of the relationships between different variables, an intersection line is also introduced in this figure. Through a comprehensive consideration of various variables, a series of structural dimension variables are determined based on crossover points between variable curves and intersection line as listed in Table 1. 


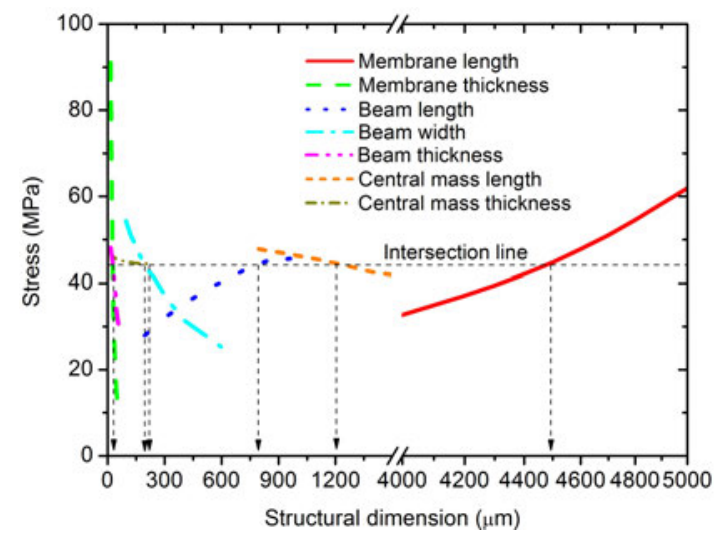

Fig. 3. Stress versus structural dimension variables.

TABLE 1. THE PARAMETERS OF THE FBBM MEMBRANE

\begin{tabular}{|c|c|c|c|c|c|c|c|}
\hline Parameter & $\boldsymbol{L}$ & $\boldsymbol{H}$ & $\boldsymbol{a}$ & $\boldsymbol{b}$ & $\boldsymbol{h}$ & $\boldsymbol{w}$ & $\boldsymbol{t}$ \\
\hline $\begin{array}{c}\text { Dimension } \\
(\mu \mathrm{m})\end{array}$ & 4500 & 30 & 800 & 200 & 30 & 1200 & 220 \\
\hline
\end{tabular}

Based on parameters of the FBBM presented in Table 1, $E$ is $1.9 \times 10^{11} \mathrm{~N} / \mathrm{m}^{2}$, and $P$ is $5 \mathrm{kPa}$. Then, the simulation results are as follows: $\sigma$ is equal to $43.6 \mathrm{MPa}$, and $\omega$ is $3.14 \mu \mathrm{m}$. Then, $Q_{1}$ and $Q_{2}$ are $1.817 \times 10^{-3}$ and $4.681 \times 10^{-4}$, respectively. Finally, the main stress and deflection equations specific to FBBM are expressed as follows:

$$
\begin{gathered}
\sigma=1.817 \times 10^{-3} \frac{P L^{293978} a^{031799}}{H^{190014} b^{03945} h^{040149} w^{021938} t^{002068}} \\
w=4.681 \times 10^{-4} \frac{P L^{507637}}{E H^{235753} a^{016714} b^{02673} h^{05443} w^{073648} t^{009719}}
\end{gathered}
$$

From Eq. (9) to Eq. (10), the stress and deflection of the FBBM regarding the structural dimension variables are clearly presented. According to the final relationships between the mechanical performance and each variable, it is beneficial to provide a guideline for designing the sensor with the FBBM structure more accurately.

\section{Performance of the sensor}

To theoretically estimate the particular values of the sensitivity of the proposed sensor, the longitudinal stress $\sigma_{x}$ and transversal stress $\sigma_{y}$ along the path are presented in Fig. 4. By comparing the results of the two sets of datas, the stress $\sigma_{x}$ is larger than $\sigma_{y}$. It is indicated that the piezoresistors implanted on the silicon substrate should be parallel to the $\mathrm{x}$ axis of the membrane. This can maximize the utilization of the transverse effect and decrease the longitudinal effect. Then, a high sensitivity and wide measurement range can be achieved. Besides, it is found that the SCRs are located at the edge between each beam and membrane pedestal side as we expected. Piezoresistors should be placed on the SCRs because more strain energy can be utilized, and therefore the piezoresistive sensitivity can be enhanced definitely. The doping concentration of the piezoresistor is depended upon implantation and annealing parameters (including boron ion dose, energy, annealing temperature, and time). In the simulation, the concentration of ion implantation is set as $3 \times 10^{14} \mathrm{~cm}^{-3}$ less than $1 \times 10^{17} \mathrm{~cm}^{-3}$, so $\pi_{44}$ can be set as $138.1 \times 10^{-11} \mathrm{~m}^{2} / \mathrm{N}\left(\right.$ or $\left.\mathrm{Pa}^{-1}\right)$ [9]. The sensitivity of the sensor for a certain mechanical pressure $P(5 \mathrm{kPa})$ and input voltage $U_{\text {in }}(5 \mathrm{~V})$ is then calculated by Eq. (11):

$$
S=\frac{\Delta U}{U_{\text {in }}} \frac{1}{P}=\frac{0.345 \times 10^{-5}\left(\sigma_{x}-\sigma_{y}\right)_{5 \mathrm{kPa}}}{V_{\text {in }}} \frac{1}{P}
$$

Eventually, the sensitivity of $4.71 \mathrm{mV} / \mathrm{V} / \mathrm{kPa}$ can be deduced.
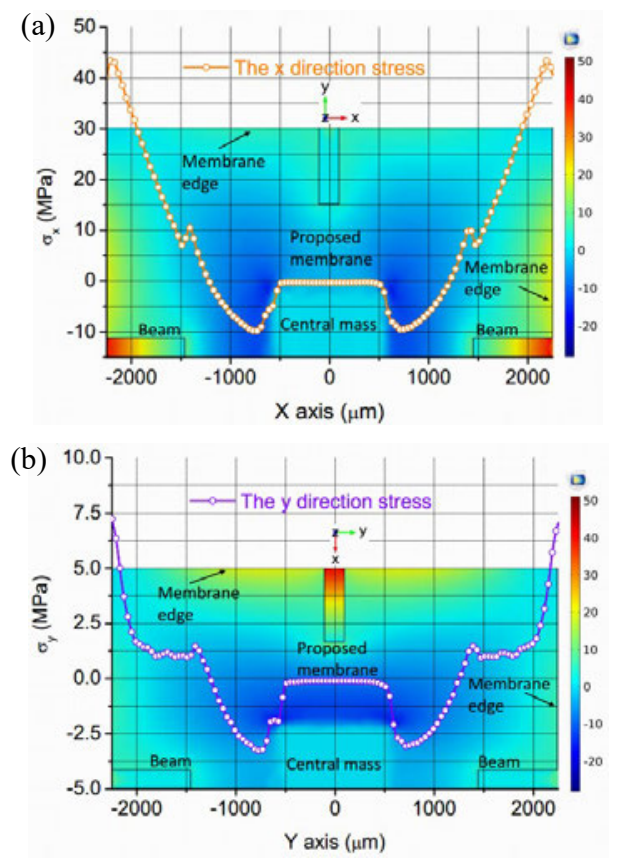

Fig.4. Stress distribution curve of the path: (a) along x-axis; (b) along y-axis.

Piezoresistive silicon pressure sensors with thin membranes have to be sensitive, accurate, and stable during pressure measurement and maintain a linear relationship between the output voltage and pressure. In certain cases, linearity is not a critical factor. For example, in case of a control switch which must indicate whether the pressure has reached a particular threshold value or not. On the contrary, some applications like level/altitude sensing using barometric sensors require a high degree of linearity. On the basis of the definition of pressure nonlinearity $(P N L)$, the relationship between $P N L$ and voltage is revealed by Eq. (12):

$$
P N L=\frac{\Delta U_{\max }}{F S S} \times 100 \%
$$

where $\Delta U_{\max }$ is the maximum output voltage difference between the real voltage output and the idealized linear output, fullscale span (FSS) is the voltage range of the full pressure, $P N L$ is the ratio of $\Delta U_{\max }$ to FSS. Finally, the pressure nonlinearity of $0.75 \%$ FSS can be deduced based on the simulation results as shown in Fig. 5. 


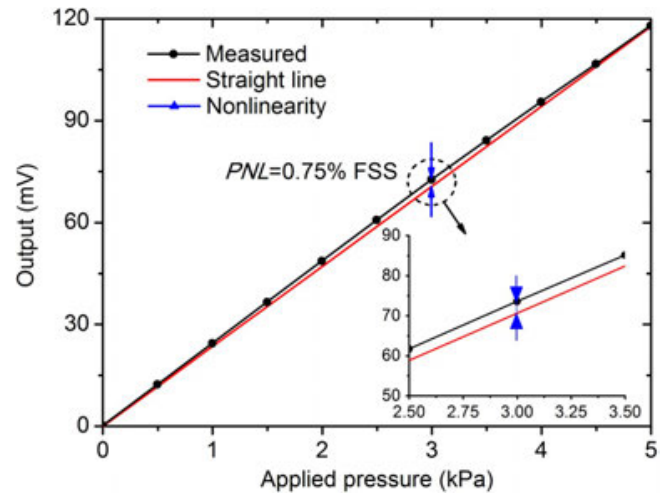

Fig. 5. Pressure nonlinearity of the FBBM structure sensor.

\section{FABRICATION PROCESSES}

Four inch $(100 \pm 0.5 \mathrm{~mm}) \mathrm{N}$-type silicon wafer oriented in the (100) direction with a resistivity of $10 \Omega \cdot \mathrm{cm}$ are used as substrate of the FBBM chip. They are $300 \pm 5 \mu \mathrm{m}$ thickness and double sides polished with a total thickness variation of $<5 \mu \mathrm{m}$. The specific fabrication processes are illustrated in Fig. 6 . The main steps are as follows:

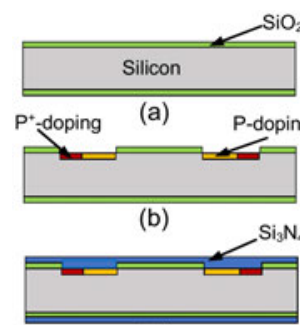

(c)

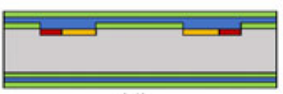

(d)

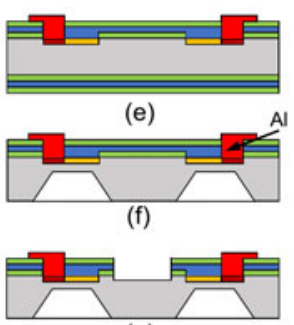

(g)

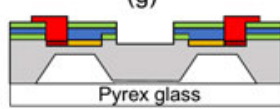

(h)
Fig. 6. Main fabrication processes used to manufacture the FBBM chip.

(a) The first step in fabrication is to deposit a $500 \pm 20 \mathrm{~nm}$ silicon oxide on both sides of the substrate by thermal oxidation. This oxide layer is used to isolate the metallization from the silicon substrate.

(b) Then, lithography is performed on the front side of silicon oxide to pattern piezoresistors. P-type resistors and heavy doping of contact regions are formed by solid source of boron thermal diffusion process.

(c) Subsequently, the low pressure chemical vapor deposition (LPCVD) is adopted to deposit the passivation layers of $\mathrm{Si}_{3} \mathrm{~N}_{4}$ to protect the piezoresistors.

(d) In the following, $\mathrm{SiO}_{2}$ layers are deposited again by the plasma enhanced chemical vapor deposition (PECVD) to further improve insulation.

(e) After that, the reactive ion etching (RIE) process is used to etch the ohm contact regions between the piezoresistors and the metal lead. The e-beam evaporation deposition is performed to sputter $\mathrm{Al}$ for the purpose of connecting of resistors and forming of bonding pads.

(f) With the objective of creating a cavity and a central mass for producing the pressure-sensitive membrane, $\mathrm{KOH}$ wet etching process is used to deeply etch the bottom of the silicon wafer. Due to anisotropy of single crystal silicon, a $54.7^{\circ}$ inclined wall is left after deep etching.

(g) Afterwards, the RIE is applied again to produce beams of the FBBM structure on the front side of the silicon wafer.

(h) In order to obtain an absolute pressure reference chamber, the bottom side of the wafer is attached to the Pyrex 7740 glass by the anodic bonding process under vacuum condition

\section{CONCLUSION}

In this study, a novel structural FBBM pressure sensor enhancing the sensitivity and linearity was proposed. A series of models for deducing the relative equations for these structures were established based on the simulation results. According to the curve fitting, the final relationships between the mechanical performance and dimension variables were determined, which could provide a guideline for designing the sensor with the FBBM structure. By altering the values in the equations, the optimized dimensions for the membrane could be found. This method was useful to design and fabricate the FBBM pressure sensor accurately. Through calculation and simulation, the proposed sensor possessed a high sensitivity of $4.71 \mathrm{mV} / \mathrm{V} / \mathrm{kPa}$ and a low pressure nonlinearity of $0.75 \% \mathrm{FSS}$ after the geometrical optimization. Finally, the main fabrication processes of the sensor chip based on bulk-micromachining and anodic bonding technology were introduced. Future work will be devoted to the interconnecting and packaging of the FBBM sensor. After that, the testing will be carried out to testify the performance of the sensor.

\section{ACKNOWLEDGMENT}

The work was supported by the China Scholarship Council for studying abroad (File No. 201508320275) and Erasmus+ project (2015-1-ES01-KA107-015460).

\section{REFERENCES}

[1] H.-D. Ngo, B. Mukhopadhyay, O. Ehrmann, and K.-D. Lang, "Advanced Liquid-Free, Piezoresistive, SOI-Based Pressure Sensors for Measurements in Harsh Environments", Sensors, vol. 15, pp. 20305-20315, August 2015.

[2] C.-Y. Lin, and J.-C. Chiou, "Design and fabrication of MEMS-based thermally-actuated image stabilizer for cell phone camera", Solid State Electron, vol. 77, pp. 64-71, May 2012.

[3] J. W. Song, J.-S. Lee, J.-E. An, and C. G. Park, "Design of a MEMS piezoresistive differential pressure sensor with small thermal hysteresis for air data modules", Rev. Sci. Instrum, vol. 86, pp. 065003, June 2015.

[4] Z. Yu, Y.L. Zhao, L. Sun, B. Tian, and Z.D. Jiang, "Incorporation of beams into bossed diaphragm for a high sensitivity and overload micro pressure sensor", Rev Sci Instrum, vol. 84, pp. 015004, January 2013.

[5] J. Albert Chiou, and S. Chen, "Pressure nonlinearity of micromachined piezoresistive pressure sensors with thin diaphragms under high residual stresses”, Sensors Actuators A Phys, vol. 147, pp. 332-339, March 2008

[6] H. Sandmaier, and K. Kuhl, "A Square-Diaphragm Piezoresistive Pressure Sensor with a Rectangular Central Boss for Low-Pressure Ranges", IEEE Trans Electron Devices, vol. 40, pp. 1754-1759, October 1993.

[7] Hsu T.-R, MEMS and Microsystems: Design, Manufacture, and Nanoscale Engineering, Wiley, 2008.

[8] W.C. Young, and R.G. Budynas, Roark's Formulas for Stress and Strain. New York: McGraw-Hill, 1986.

[9] O. N. Tufte, and E. L. Stelzer, "Piezoresistive Properties of Silicon Diffused Layers”, J. Appl. Phys, vol. 34, pp. 313-318, September 1963. 